Revue des patrimoines

\title{
Une découverte majeure en Gironde, le fonds du carrossier Bellion, archives et matériels
}

\author{
Sylvie Termignon
}

\section{OpenEdition}

Journals

Édition électronique

URL : http://journals.openedition.org/insitu/11921

DOI : 10.4000/insitu. 11921

ISSN : 1630-7305

Éditeur

Ministère de la culture

Référence électronique

Sylvie Termignon, « Une découverte majeure en Gironde, le fonds du carrossier Bellion, archives et matériels », In Situ [En ligne], 27 | 2015, mis en ligne le 02 novembre 2015, consulté le 21 avril 2019 URL : http://journals.openedition.org/insitu/11921 ; DOI : 10.4000/insitu.11921

Ce document a été généré automatiquement le 21 avril 2019

\section{(c) $($ i) $(9)$}

In Situ Revues des patrimoines est mis à disposition selon les termes de la licence Creative Commons Attribution - Pas d'Utilisation Commerciale - Pas de Modification 4.0 International. 


\title{
Une découverte majeure en Gironde, le fonds du carrossier Bellion, archives et matériels
}

\author{
Sylvie Termignon
}

\section{Introduction}

1 La découverte de l'entreprise de carrosserie Bellion est liée à l'histoire du musée de la Voiture à cheval ${ }^{1}$.

2 Ce musée municipal qui bénéficie d'une appellation «musée de France » est situé dans le parc de la citadelle, à Bourg, dans le département de la Gironde. De la forteresse militaire, il reste les vestiges souterrains et il est difficile pour le visiteur non averti d'imaginer une place forte datant $d u x \mathrm{XII}^{\mathrm{e}}$ siècle quand se déploie sous ses yeux un cadre qui rappelle plutôt le XVIII ${ }^{\mathrm{e}}$ siècle avec sa chartreuse et ses jardins à la française. Le musée a ouvert ses portes le $1^{\mathrm{er}}$ avril 1995. Il est installé dans les anciens chais du XVIII siècle qui ont été agrandis et aménagés pour l'occasion. Les voitures exposées dans le musée sont essentiellement régionales. Elles ont toutes été, soit fabriquées, soit utilisées en Aquitaine entre le XIX ${ }^{e}$ siècle et le début du XXe siècle.

3 L'une des particularités du musée de la Voiture à cheval vient de ce que les collections ne sont pas documentées. On remarque cependant, sur les bouchons des essieux de certaines voitures, la signature ou le nom du carrossier ainsi que la ville où il exerce sa profession. À partir de ces inscriptions relevées sur les véhicules, en 1999, un programme de recherche a été mis en place. La recherche a été menée au service des archives municipales de Bordeaux et aux archives départementales de Gironde. En effet, pour une collection composée actuellement de quarante-cinq voitures hippomobiles, nous comptons treize carrossiers bordelais, deux carrossiers de Blaye, deux de Libourne et un de Gauriac, dans le canton de Bourg.

Parmi eux, nous trouvons Guillemot, qui a réalisé un demi-tonneau exposé aujourd'hui au musée. Il travaillait rue du Château d'eau à Bordeaux, entre 1900 et 1935. Le sellier 
carrossier Desjacques, de Libourne est également représenté dans les collections du musée. Ce sont là deux carrossiers de province méconnus mais qui ont toute leur importance dans le cadre de cette étude.

\section{Le programme de recherche}

$5 \quad$ L'étude a porté dans un premier temps sur les annuaires du Sud-Ouest, afin de dater non pas les voitures mais plutôt les entreprises qui les ont construites. La datation des entreprises reste cependant approximative. Chacun peut créer sa société à une date donnée et ne vouloir figurer dans l'annuaire que quelques années plus tard. Cela permet toutefois en premier lieu de délimiter précisément la chronologie de ces sociétés. Par la suite, la recherche pourra être affinée.

6 Les annuaires du Sud-Ouest ont été patiemment dépouillés, année après année. Je me suis attachée à savoir si mes carrossiers avaient traversé la Première, puis la Seconde Guerre mondiale. Certains ont continué à travailler jusqu'après 1955. Je réalise que ce patrimoine, sur l'échelle du temps, est très proche de nous. Je décide alors de noter sans distinction tous les noms des carrossiers, charrons, forgerons, selliers, bourreliers... pour les villes de Bordeaux, Libourne et pour le canton de Bourg. Ce relevé établi, je téléphone à toutes les personnes de ces noms habitant ces villes pour savoir si, par hasard, elles ne descendent pas d'une famille de charron, forgeron ou autre...

7 C'est ainsi qu'à Libourne, je prends contact avec Michel Bellion qui me dit tout naturellement descendre d'une famille de charrons. Il vit encore dans la maison de famille et possède toujours la forge, en état de marche. Il m'invite à venir chez lui et me montre dès mon arrivée un catalogue présentant des voitures hippomobiles : je n'imagine pas alors la somme de documents relatifs à cette entreprise, classés dans un coin de la maison. J'ai tellement plus l'habitude de rencontrer des personnes attachées aux objets plutôt qu'aux archives... De plus, je n'avais jamais vu de forge auparavant et comme je me montre intéressée par cet outil, M. Bellion la fait fonctionner en actionnant le soufflet, dans cet atelier où tout est resté en place depuis la fermeture de l'entreprise en 1970.

8 Lors de nos divers échanges, Michel Bellion montre un attachement presque viscéral à ce qui représente ses racines. Plus tard, je me contenterai de lui dire que si sa famille ne se montre pas intéressée par ce patrimoine ou si, plus ennuyeux parce que plus douloureux, elle ne peut pas conserver ce fonds, il pourra le verser au musée de la Voiture à cheval. Mais je n'insiste pas davantage, compte tenu de l'importance que revêt cet atelier familial pour Michel Bellion.

9 Michel Bellion décède le 28 juin 2011 à l'âge de 77 ans ; dans un petit répertoire, sa fille Michèle trouve mon prénom ainsi que mon numéro de téléphone. Comme son père, Michèle Bellion est très attachée à ce patrimoine familial. Pour elle, il est évident qu'il doit être préservé, elle convainc donc sa famille d'en faire don au musée de la Voiture à cheval (fig. 1). 


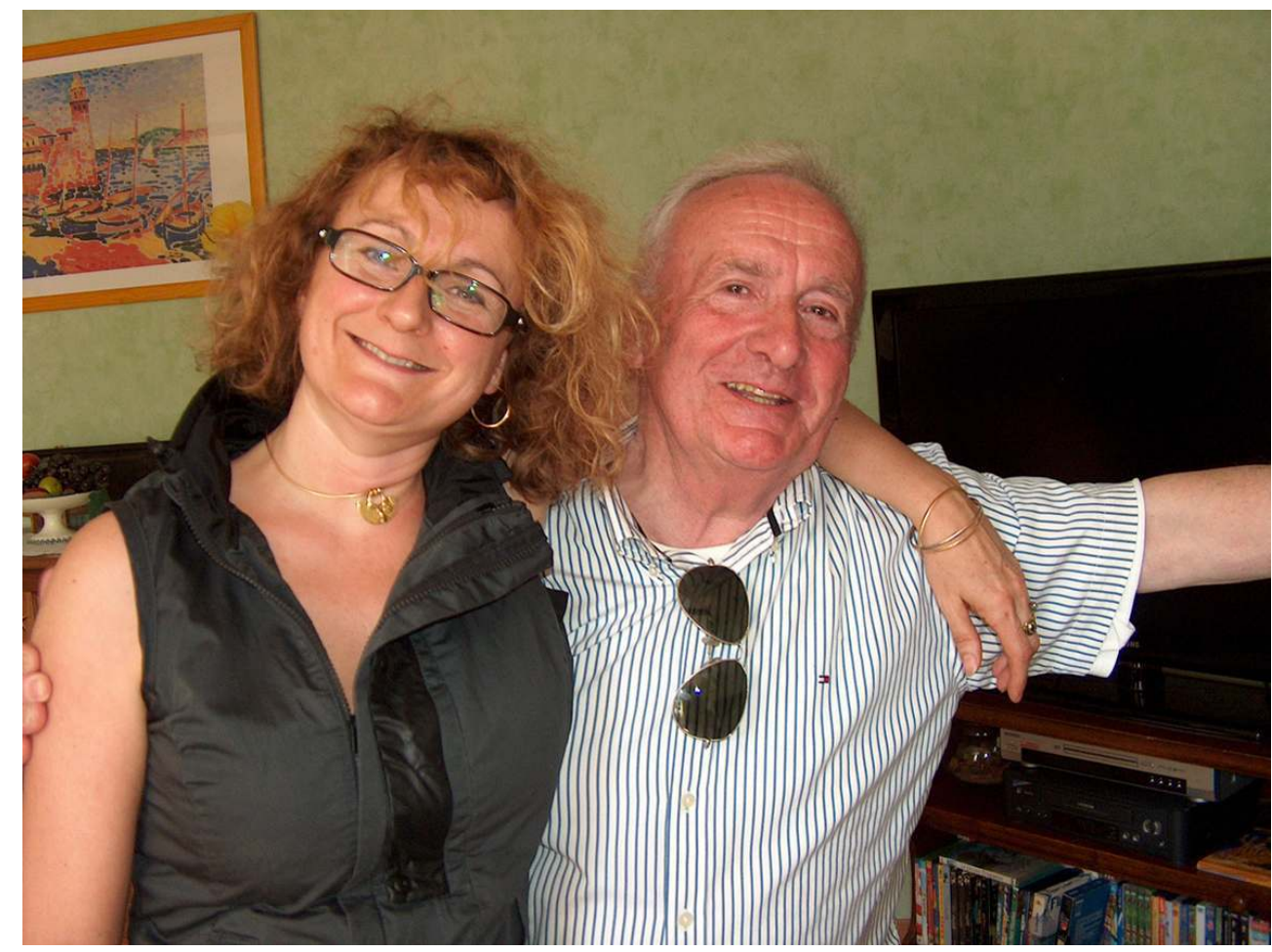

Michèle et Michel Bellion.

Phot. Sylvie Termignon. ( ) Sylvie Termignon.

10 Pour mieux comprendre ce qu'est cet atelier, il est nécessaire de donner quelques repères chronologiques et d'observer son évolution, ses mutations. L'atelier Bellion ouvre ses portes en 1874. Les carrossiers travaillent à la construction de voitures hippomobiles. Puis progressivement, ils se mettent à fabriquer des voitures automobiles et des bicyclettes. Petit à petit, l'atelier se reconvertit en entreprise de menuiserie qui ferme ses portes en 1970, date de la fin d'activité de la société Bellion.

11 Après le décès de Michel Bellion, la maison n'a pas été conservée par la famille et Michèle Bellion, sa fille, souhaite la vider, ainsi que l'atelier, avant de vendre le tout. Il faut récupérer d'une part la forge, d'autre part les outils et enfin, la documentation.

\section{Collecte de données}

Sur le terrain, accompagnée de mon collègue Philippe Pinier ainsi que de deux bénévoles, Angelina Jacquin et Gilles Robert, nous commençons par effectuer des clichés photographiques des parties qui nous semblent importantes ainsi que des détails, y compris ceux qui nous paraissent insignifiants. Après la vente du bâtiment, nous n'aurons plus l'occasion de revenir ; tout aura été démonté, l'atelier totalement vidé. Il nous faut par conséquent être le plus exhaustif possible.

13 Pour cette collecte, il est nécessaire de se projeter dans le futur. Puisque nous travaillons pour un "musée de France", nous allons devoir passer devant la commission interrégionale d'acquisition en proposant une liste d'objets à inscrire sur l'inventaire ainsi qu'une liste d'objets d'étude. Il faut également concevoir un projet scientifique et 
culturel pour assurer, d'une part, la conservation, mais également la diffusion auprès du public : «Qu'allons-nous faire de ce patrimoine? ? est la question que nous devons nous poser en amont.

Nous nous sommes interrogés également sur le fonctionnement de la forge. In situ, nous trouvons un foyer sous une hotte. Sur le côté gauche trône un imposant soufflet. Pour la construction du foyer, nous notons la présence de briques réfractaires posées sur un squelette de métal, le tout entièrement recouvert de plâtre. Nous nous sommes évertués à vider le foyer pour en découvrir les éléments constitutifs. Ainsi, sous la cendre, nous remarquons un lit de sable utilisé sans doute pour son inertie thermique.

En dégageant l'intérieur du foyer, nous avons retrouvé sur le côté gauche la tuyère branchée au soufflet (fig. 2). Cependant, sur le côté droit, nous remarquons la présence d'une deuxième tuyère. En prenant un peu de recul, nous repérons les traces d'une hotte plus ancienne qui devait être de forme pyramidale. D'autre part, il y a près de cette hotte des points d'ancrage pour un second soufflet. Nous avons retrouvé également dans l'atelier le berceau d'un soufflet plus petit.

Figure 2

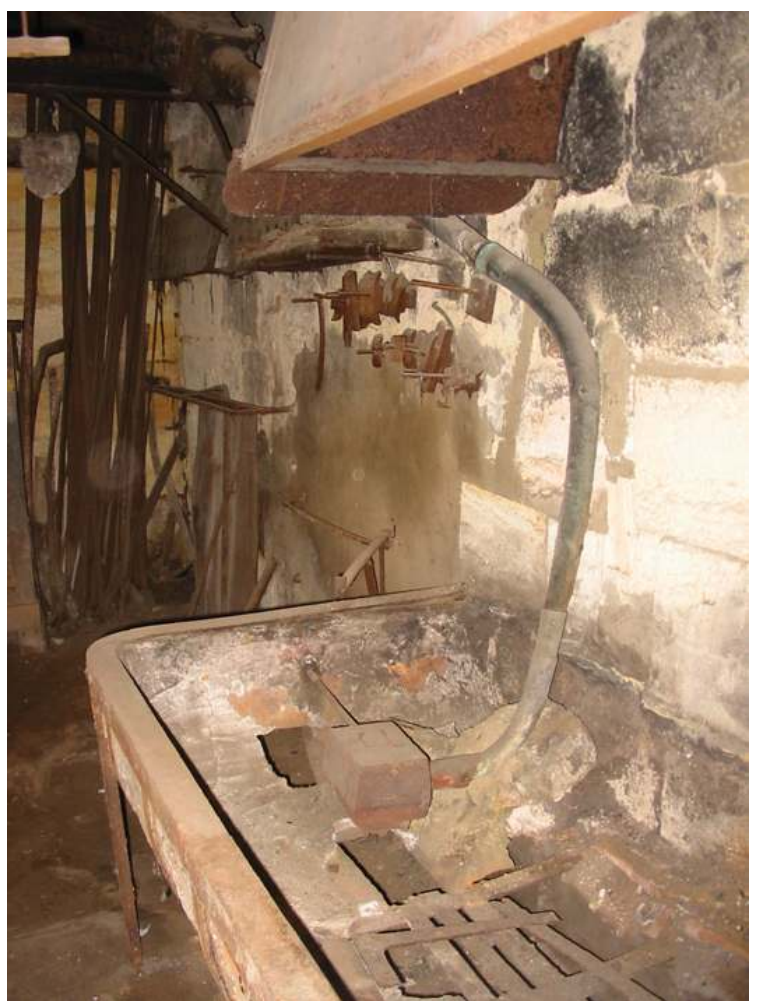

Foyer et sa tuyère.

Phot. Sylvie Termignon. @ Sylvie Termignon.

Dans l'Art du serrurier, rédigé en 1826 par Hoyau², la forge décrite comme idéale possède deux soufflets, un petit qui permet de porter au feu de petits éléments et un plus important pour travailler sur des objets plus volumineux; il est également possible de relier les deux soufflets sur une même tuyère pour forger des objets de grand volume. Le soufflet est à deux vents, ce qui est plus pertinent pour le forgeron car le souffle est continu, permettant de maintenir le fer à température haute et constante. 

afin d'en retenir ses caractéristiques; les divers points d'ancrage nous permettront, éventuellement, de reproduire cet espace dans le cadre du musée. Parmi les éléments de la forge, l'enclume de 156 kilos, achetée en 1904, provient des entreprises Henri Gallinié et successeurs de Bordeaux.

urant nos manipulations, nous nous apercevons rapidement qu'il va nous falloir faire du tri, puisque parmi les outils en lien avec la profession, nous trouvons également des objets sans rapport avec la carrosserie. En effet, on peut imaginer que depuis 1970, le lieu a servi d'espace de stockage à la famille. Parmi les outils, nous inventorions entre autre des étampes et contre-étampes qui servent à donner des formes particulières au fer. Nous avons également repéré les clefs de charron qui servent à démonter les roues des voitures, les pinces et tous les outils se rapportant au métier de carrossier encore présents dans l'atelier (fig. 3).

Figure 3

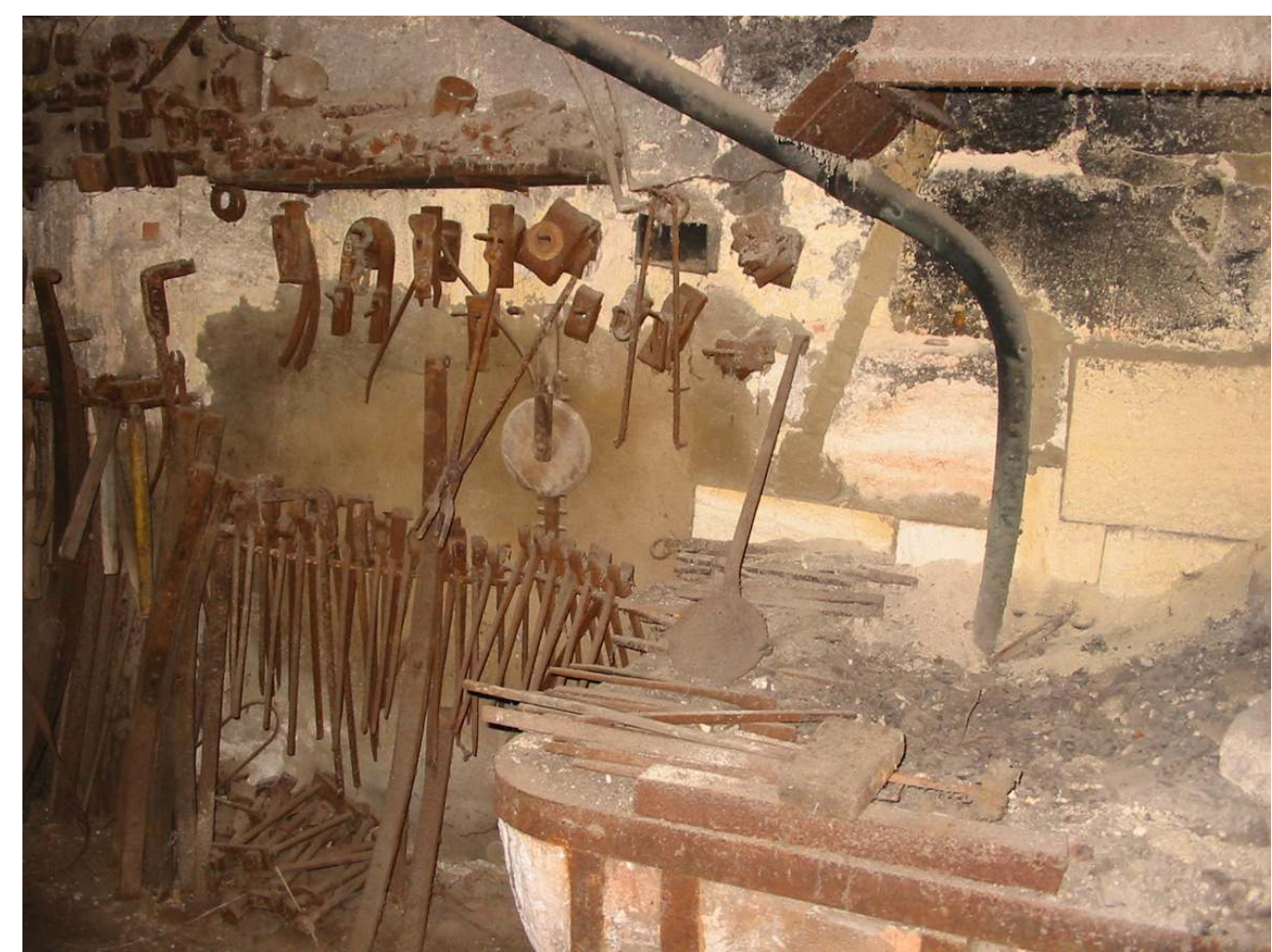

Les outils dans l'atelier lors de leur découverte.

Phot. Sylvie Termignon. (c) Sylvie Termignon.

Parmi les accessoires, nous découvrons des poignées et contre-poignées de portes, des boutons de diverses couleurs pour capitonner les sièges, des boulons à quatre pans, des plaques avec les marques du fabricant, un essieu patent à graisse avec boîtes et écrou, un essieu patent à huile signé « 655 Binder à Paris » avec boîtes et chapeaux, un moyeu en cours de réalisation, une roue en bois réalisée par un apprenti, des œillets, des contreœillets et autres petits objets de garniture dans leurs boîtes d'origine rangées dans des casiers créés à cet effet. Cet atelier, conservé avec respect par Michel Bellion jusque dans son organisation, nous semble suspendu dans le temps. 

voitures automobiles. Nous avons décidé de ne pas scinder ce fonds pour respecter la continuité de l'atelier. Une autre partie concerne également la menuiserie. Ces documents, qui n'intéressent pas directement le musée, seront versés au service des archives départementales de Gironde.

21 facturiers d'entreprise, de carnets de commandes clients et de la liste nominative des six employés qui se sont succédé dans l'atelier. Dans les registres de correspondance dont voici deux extraits, nous retrouvons Guillemot, carrossier à Bordeaux, dont un demitonneau est exposé au musée de la Voiture à cheval de Bourg (fig. 4).

Figure 4

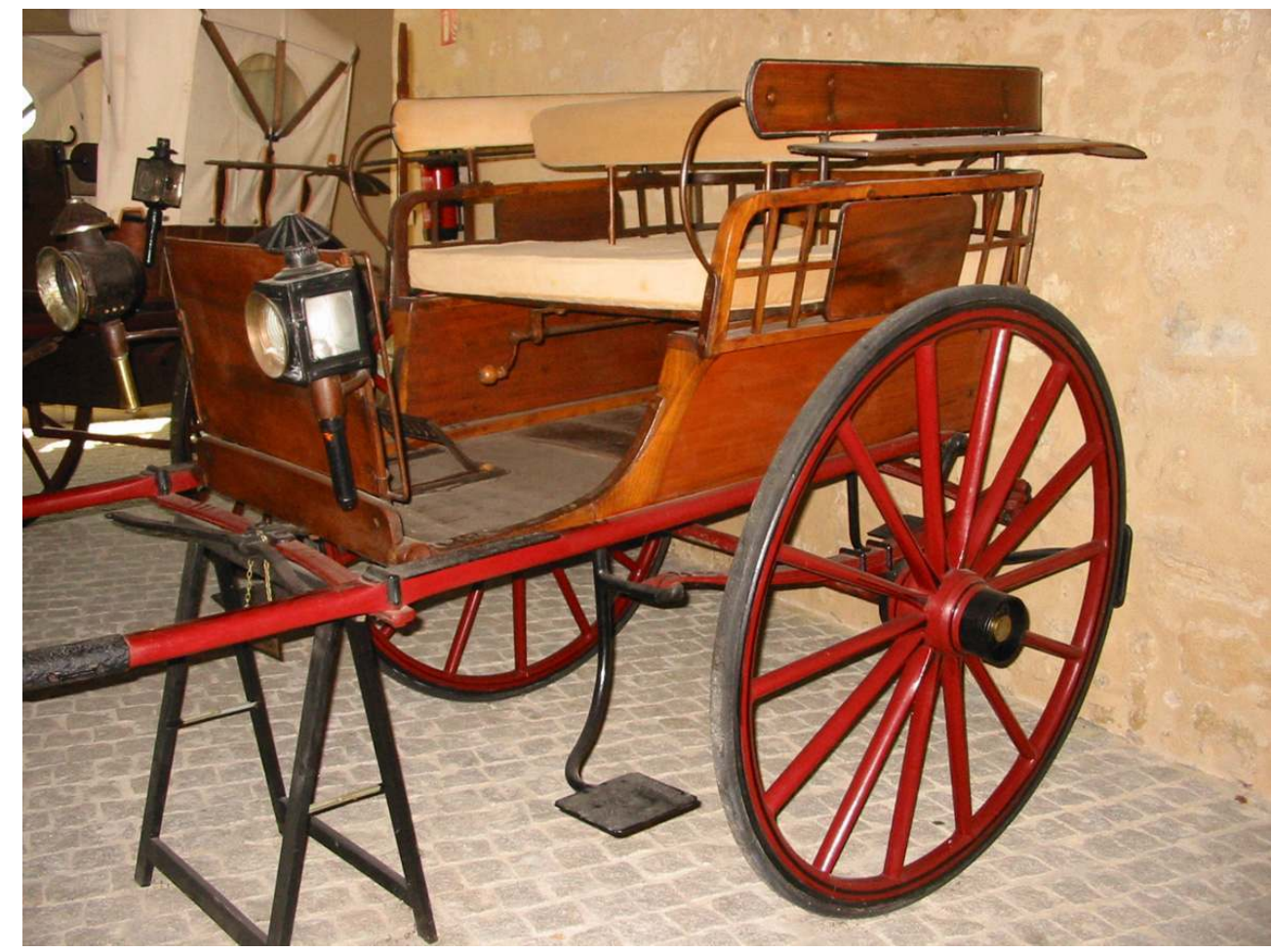

Demi-tonneau carrossé par Guillemot à Bordeaux. Musée de la Voiture à cheval de Bourg.

Phot. Sylvie Termignon. (c) Sylvie Termignon.

Il écrit à Bellion, carrossier à Libourne, le 5 mai 1902 :

Avec la meilleure volonté, je ne puis vous satisfaire. J'ai envoyé votre carte postale au forgeron, car la calèche n'est plus à moi. Il m'a répondu que la caisse de l'omnibus était faite, mais je crois que si vous lui écrivez, vous pourrez réussir à lui acheter la caisse, car il la démolira et il serait plus favorable qu'il vous la vende. Je vous donne son adresse.

M. Fort, forgeron, rue de Kater $\mathrm{N}^{\circ} 18$ Bordeaux.

Et si vous venez me voir à Bordeaux et je vous mènerai chez lui. Je suis toujours prêt à vendre le coupé Binder.

Dans un second courrier, Guillemot écrit à Bellion, le 13 mai 1902 :

Si vous voulez acheter la caisse de la calèche elle est chez le forgeron depuis aujourd'hui. Je leur ai donné votre lettre, je crois qu'ils veulent 80 frs de la caisse, avec le siège et les marchepieds. Je crois que vous ferez mieux de venir et au sure moi je ferais mon possible pour vous la faire laisser le meilleur marché et je vous 
vendrais le vieux train qui est chez moi ou l'on pourra y adapter la caisse pour la conduire par route.

24 Ces courriers sont intéressants car ils nous incitent à nous interroger sur «l'authenticité » des voitures que nous avons dans nos collections, tant privées que publiques. La construction des voitures s'inscrit dans une démarche dans laquelle les carrossiers recyclent parfois les véhicules de leurs confrères.

Les échanges favorisent l'enrichissement de nos connaissances sur certaines pièces de nos collections. C'est le cas aujourd'hui pour le musée de la Voiture à cheval puisqu'au moins deux carrossiers, Guillemot et Desjacques, dont des voitures font partie des collections du musée, sont cités dans ces correspondances.

Il est également judicieux d'énumérer la quantité de registres et les périodes couvertes, qui vont nous donner des pistes pour les futures recherches. Dans le détail, nous comptons cinq registres de correspondance, allant de 1880 à 1919. Parmi la documentation, divers catalogues ou publicités fournisseurs s'échelonnent de 1913 à 1934, alors que cinq livres de comptes-paiements des fournisseurs vont de 1883 à 1937. Les registres facturiers (vingt-deux) sont ouverts en 1880 et sont clos en 1934. Les quatre registres de commandes débutent avec l'ouverture de l'atelier en 1874, la dernière année mentionnée est 1931. Dans le fonds documentaire, nous recensons un répertoire client mais également des papiers relatifs à la gestion du ménage Bellion entre 1883 et 1952 .

Nous constatons quelques manques dans ce fonds; cependant, son inventaire précis nous permettra de comprendre pour certaines périodes le mode de fonctionnement d'une société de carrosserie provinciale. Notamment, les facturiers nous donnent des indications sur les échanges et le rayonnement géographique de la société Bellion avec d'autres fournisseurs. C'est en Normandie qu'il va, par exemple, se procurer les tissus, les couleurs et les vernis. Le verre à vitre ainsi que le combustible sont commandés à Bordeaux. Il commande aussi à Paris des éléments de carrosserie tels que ceux que nous avons énumérés dans la liste d'objets mentionnée un peu plus haut. Cela peut paraître anecdotique, mais les papiers relatifs à la gestion du ménage permettent d'aborder la vie quotidienne ainsi que ses changements durant les deux grandes guerres pour cette famille d'une petite ville de province.

D'autre part, l'importance de ce fonds documentaire dépasse le cadre du musée municipal de Bourg et devrait favoriser l'enrichissement des collections du musée national de la Voiture et du Tourisme à Compiègne. En effet, ce musée possède dans ses collections une voiture carrossée par Bellion. Il s'agit de la voiture d'un dentiste ambulant inventeur de l'eau galvanique qui se nomme Sorino. Ainsi, la consultation du répertoire clients ainsi que des registres de commandes qui couvrent une période allant de 1874 à 1931 nous permettra peut-être de dater plus précisément ce véhicule.

\section{Conclusion}

29 Cette découverte a toute son importance pour le musée de la Voiture à cheval de Bourg, car elle participe non seulement à l'enrichissement du fonds documentaire mais également matériel de ce dernier. Elle est en adéquation avec la thématique du musée qui présente des voitures hippomobiles régionales, datant de la fin du XIX ${ }^{\mathrm{e}}$ siècle et du début du XX ${ }^{\mathrm{e}}$ siècle. 

province. Ces derniers, peu connus, sont peu étudiés; ils figurent pourtant dans nos collections, comme au musée national de la Voiture et du Tourisme de Compiègne, et ont, de par les exigences de leurs commanditaires, participé à l'évolution technique dans la construction des voitures.

\section{NOTES}

1. - Adresse du musée de la Voiture à cheval : 33710 Bourg.

2. - BURY, architecte et HOYAU, ingénieur mécanicien. "Abrégé de l'Art du Serrurier ». Dans Modèles de Serrurerie, choisis parmi ce que Paris offre de plus remarquable sous le Rapport de la Forme, de la Décoration et de la Sureté; accompagnés des Détails qui doivent en faciliter l'exécution. Paris : Bance Aîné éditeur, 1826, p. 2-3.

\section{RÉSUMÉS}

En 1874, Louis Bellion, s'installe à Libourne et fonde son atelier de carrossier. La collection Bellion retrace l'histoire d'une famille profondément attachée à son passé, à son activité et qui a préservé ses archives ainsi que son matériel, de la fondation de cet atelier à nos jours. Elle est, aujourd'hui, l'une des collections les plus complètes de Gironde, constituée de ses archives mais également de sa forge avec ses outils. Une première approche de ce fonds qui doit aujourd'hui intégrer le musée de la Voiture à cheval à Bourg en Gironde permet d'aborder le tissu socioprofessionnel de cette famille de carrossiers libournais dans une période de transition, où les automobiles prennent le pas sur les voitures hippomobiles. C'est un exemple de collecte ethnographique qui permet de documenter quelques voitures qui sont aujourd'hui au musée de la Voiture à cheval ainsi qu'au musée national de la Voiture et du Tourisme à Compiègne.

A major discovery in the Gironde department, the Bellion collection of a coach-building family. In 1874, Louis Bellion settled in Libourne and established his coach-builder's firm. The collection that this firm has left today tells the story of a family deeply attached to its past and to its 
profession. The firm has kept all its archives and equipment from the creation of the works up to the present day. It is one of the most complete collections in the Gironde, comprising not only the family archives but also its forge with all its tools. A preliminary study of this collection which is destined to become part of the museum of horse-drawn vehicles at Bourg, in the Gironde, allows for a better understanding of the social and professional aspects of this coachbuilder's family in Libourne, during a period which saw the progressive replacement of horsedrawn vehicles by motor cars. It is a collection of ethnographic interest that also gives documentation about some of the vehicles held at the museum of the horse-drawn carriage as well as at the national museum of carriages and tourism, in Compiègne.

INDEX

Keywords : coach-builder, Bellion, archives, musée de la Voiture à cheval, Bourg, musée national de la Voiture et du Tourisme, Compiègne, Libourne, smithy, Bordeaux

Mots-clés : carrossier, Bellion, archives, musée de la Voiture à cheval, Bourg, musée national de la Voiture et du Tourisme, Compiègne, Libourne, forge, Bordeaux

\section{AUTEUR}

\section{SYLVIE TERMIGNON}

conservateur délégué des antiquités et objets d'art (Gironde). Responsable du musée de la Voiture à cheval sylvietermignon@gmail.com 